group can take over 500 steps on the same track before falling off.

There is no control over the direction in which the side chain steps along the track and the system simply equilibrates to give the most thermodynamically favourable distribution of nitrostyrene groups. Nevertheless, using a track with five amine stations Leigh and co-workers were able to show that a side chain could migrate from one end to the other and, in doing so, perform a rudimentary task, namely quenching a fluorescent group.

\section{SURFACE CHEMISTRY}

\section{Panning for gold}

\section{J.Am. Chem. Soc. http://doi.org/hvr (2012)}

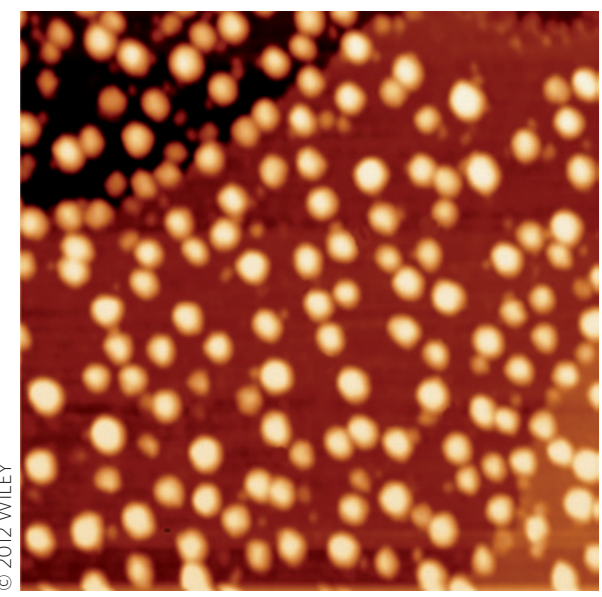

Many catalysts consist of active metal species adsorbed on an oxide surface, and they need to be highly dispersed to maintain activity. Unfortunately, metal atoms have a tendency to group into larger, less reactive particles, which has led to several attempts at reversing this process, but the mechanisms by which they worked were not understood. Now, Bing Yang from the Fritz Haber Institute in Berlin and colleagues have found that molecules of tris(phenylthio)benzene (TPB) are capable of trapping individual atoms of gold on either a gold or alumina surface.

Using scanning tunnelling microscopy, Yang and colleagues observed adsorbed gold atoms disappearing from the metal's surface as they coordinated to TPB ligands. This trapping of adatoms occurred with varying configurations of the TPB molecules, and so anything up to six atoms could become attached to the ligands. The team discovered that below $100 \mathrm{~K}$, a flat-lying, single molecule of TPB bound to six gold atoms was stable. With TPB dimers the incorporation of six atoms was seen at room temperature.

When Yang and colleagues applied TPB to gold aggregates on an alumina surface, modelling a real catalyst, they observed several new aggregates, only $15 \AA$ in size. The ability of TPB to trap gold atoms on both a gold and an oxide surface demonstrates its potential as a redispersion agent. Further research would need to be done to determine how to remove the ligand molecules from the surface after use, so as to avoid poisoning the catalyst. Yang and colleagues suggest photochemical bleaching or ozone etching as potential routes to do this.

$H P$

\section{ORGANIC SYNTHESIS}

\section{Catalytic compatibility}

Science 335, 1471-1474 (2012)

Diazomethane $\left(\mathrm{CH}_{2} \mathrm{~N}_{2}\right)$ is widely used in organic synthesis, for example as a reagent in esterification, dipolar cycloaddition and epoxidation. However, the use of diazomethane is limited by its toxicity and explosiveness, and even its precursors are unsafe.

Now, Bill Morandi and Erick Carreira from the Laboratory of Organic Chemistry in Zürich have developed a reaction in which diazomethane is formed in situ and then consumed by a metal catalyst, which avoids significant build-up of it, removing the potential hazard. A catalyst for this reaction has to be made compatible with the strongly oxidizing and strongly alkaline aqueous conditions in which the diazomethane is formed. The team tested catalysts formed by a range of transition metals and the highest yields were obtained with an iron porphyrin, which is non-toxic and inexpensive.

In Morandi and Carreira's method, diazomethane is generated in the aqueous phase and migrates to the organic phase, where it reacts with the catalyst. Separating the catalyst from the harsh conditions required to generate diazomethane means it resists decomposition. The researchers achieved up to $89 \%$ yield for the cyclopropanation of a range of alkenes using the iron porphyrin catalyst. A watersoluble rhodium catalyst gave no product, but a hydrophobic one resulted in $45 \%$ yield, demonstrating the importance of the catalyst being compatible with the organic phase. HP

Written by Stuart Cantrill, Stephen Davey,

AnnePichon and Heather Powell

\section{Correction}

In the Research Highlight 'Force microscopy takes charge' (N. Withers Nature Chem. 4, 336; 2012), Fabian Mohn's surname was spelled incorrectly. Corrected in the HTML and PDF versions after print, 26 April 2012.

\section{blog $_{\text {roll }}$ 空}

\section{Space dinosaurs!}

Highly respected organic chemist publishes a Perspective in JACS, chaos ensues.

Ronald Breslow - past president of the ACS and Priestley medallist in 1999 - is a "well-respected research lion", in the words of SeeArOh at Just Like Cooking (http://go.nature.com/xO3kep). Few would dispute his place in the chemistry firmament. With many achievements behind him, surely he has earned the right to close a Perspective article in JACS (http://go.nature.com/i5DyA2) on the possible origin of homochirality in biological molecules with a chuckle - suggesting that humans would be better off not meeting the advanced dinosaurs, with amino acids of the opposite chirality, who may populate distant corners of the universe!

Apparently not. At least, not according to some of the dinosaur-focused bloggers/ newshounds out there - such as Dinosaur Tracking (http://go.nature.com/tB19fD) or Discovery (http://go.nature.com/g31ylj). In their defence, however, they were reacting to the press briefing that the ACS somewhat mystifyingly released. It was mystifying because it ignored the six pages of discussion about the origin of chirality of amino acids to focus on the last two 'space dino' sentences.

The combination of Breslow and space dinos proved tempting for some chemistry bloggers, with SeeArrOh covering the paper and its final words in the goodhumoured tone in which they were surely intended. Paul Bracher, who blogs at ChemBark (http://go.nature.com/ZibD8I), has worked in the origin-of-life field, and actually discussed the scientific content of the paper - how refreshing! But Bracher certainly doesn't mince his words giving his opinion of the ACS press office.

The final twist in the dinosaur's tale came in the comments on Just Like Cooking and ChemBark: hasn't Breslow written something quite similar in Tetrahedron Letters (http://doi.org/bs5wzc) and the Israel Journal of Chemistry (http://doi.org/ csf7xr)? The similarity is such that, at the time of writing, JACS has removed the paper from its website, citing copyright concerns. 